\title{
Core Topics in Transesophageal Echocardiography
}

\author{
Robert Feneck, John Kneeshaw, Marco Ranucci. Cambridge University Press, 2010. \\ ISBN 978-0-521-73161-4
}

\author{
A. Stéphane Lambert, MD
}

Received: 24 May 2011 / Accepted: 7 June 2011 / Published online: 17 June 2011

(C) Canadian Anesthesiologists' Society 2011

This reference book on transesophageal echocardiography (TEE) includes contributions from TEE practitioners (anesthesiology and cardiology) from across Europe. The stated intent of this book is to provide a "reference that would cover the necessary topics required for the accreditation exams, but will also help the reader in daily TEE practice". The overall format is more compact than a standard textbook, yet will not fit in the pocket of a lab coat. The book contains roughly 400 pages, organized in 24 chapters and divided into 2 major sections. Section 1 covers basic TEE knowledge, such as physics and Doppler principles, indications, training and safety, left ventricular and right ventricular assessment, valves, masses, and aorta. Section 2 deals with specific clinical circumstances that the TEE practitioner encounters every day, e.g., surgery for coronary revascularization and valvular and congenital surgery.

As the title of the book (Core Topics) implies, the goal is to focus on the essentials and to dispense with the trivia. As a result, the information in the book tends to be presented in a concise fashion. Still, the basic TEE facts are accurate and reasonably thorough. Also, most chapters are generally well-referenced. In my view, some concepts would benefit from more detailed explanations but, as with many things in education, "clarity is in the eye of the beholder", and different readers prefer different things.

The book is visually rich and contains multiple drawings, illustrations, and TEE images to complement the text.

The TEE images are of good resolution and clarity, and the graphic illustrations also tend to be clear and to the point. I note that some drawings resemble well-known

A. S. Lambert, MD $(\bowtie)$

University of Ottawa Heart Institute, Ottawa, ON, Canada

e-mail: slambert@ottawaheart.ca previously published images and could be better referenced. Others, like the drawings of the standard TEE views from the 1999 American Society of Echocardiography/ Society of Cardiovascular Anesthesiologists (ASE/SCA) Guidelines, are used throughout the book but are referenced only once. In such a heavily illustrated book, legends play an important part in the overall learning experience. The legends in some chapters tend to be very short and more details would be beneficial, like the TEE view depicted on the image.

Finally, many of the TEE images would benefit from added labels to emphasize the teaching points.

A linked website contains the videos referenced in the book, and most TEE images in the book have corresponding videos on the website. The website was somewhat disappointing: the videos are small and they cannot be stopped once started unless the page is refreshed. There is no means to freeze the video or to go back and forth between frames. Many videos do not include an electrocardiogram and none of them appears to have labels. In this day and age, when videos can be edited easily at home on a personal computer, this falls a bit short of expectations. Finally, some of the video loops play very fast, and it may be difficult for an untrained eye to appreciate the teaching points. On the positive side, many videos have better legends than the corresponding figures in the book. The good news: a website format (rather than a DVD accompanying a book) will allow the editors/publishers to remedy most of these shortfalls by modifying the interface and uploading edited videos.

Overall, this is an nice textbook that covers most of the basic TEE curriculum. I suspect it was created in response to a need for a European-based TEE reference book. In North America, however, it joins an already relatively saturated market. In format and content, it is in direct 
competition with several other popular "essentials" books that cater to a similar audience and cover similar material in a similar fashion. Finally, in an age of interactive DVDs and iPhone apps, this book probably suffers mostly from the fact that it is... a book. In 2011, many physicians are more likely to carry tablets rather than books into the operating room. Still, if you're new to the field of TEE and you are looking for a basic text to get you started, this book is definitely an option.

Competing interest None declared. 